Highly Energetic Physical Processes and

Mechanisms for Emission from Astrophysical Plasmas

IAU Symposium, Vol. 195, 2000

P. C. H. Martens, S. Tsuruta, and M. A. Weber, eds.

\title{
Plasma Masers in Radio Pulsars
}

\author{
M. Lyutikov ${ }^{1}$ and R. Blandford \\ Canadian Institute for Theoretical Astrophysics, 60 St. George Street, \\ Toronto, Canada \\ G. Machabeli \\ Abastumani Astrophysics Observatory, A. Kazbegi Av. 2a, Tbilisi \\ 380060, Republic of Georgia
}

\begin{abstract}
.
Relativistic plasma masers operating on the anomalous cyclotronCherenkov resonance $\left(\omega-k_{\|} v_{\|}+\omega_{B} / \gamma_{\text {res }}=0\right)$ and on the Cherenkovdrift resonance $\left(\omega-k_{\|} v_{\|}-k_{x} u_{d}=0\right)$ are capable of explaining the main observational characteristics of pulsar radio emission. Both electromagnetic instabilities are due to the interaction of the fast particles of the primary beam and from the tail of the distribution with the normal modes of a strongly magnetized, one-dimensional electron-positron plasma. In a typical pulsar, both resonances occur in the outer parts of the magnetosphere at $r_{\text {res }} \approx 10^{9} \mathrm{~cm}$.
\end{abstract}

We have shown (Lyutikov, Blandford, \& Machabeli 1999a) that pulsar radiation may be generated by two kinds of electromagnetic plasma instabilitiesthe cyclotron-Cherenkov instability, developing at the anomalous Doppler resonance $\left(\omega(\mathbf{k})-k_{\|} V_{\|}+\omega_{B} / \gamma_{\text {res }}=0\right)$, and the Cherenkov-drift instability, developing at the Cherenkov-drift resonance $\left(\omega(\mathbf{k})-k_{\|} V_{\|}-k_{x} u_{d}=0\right)$. The cyclotronCherenkov instability is responsible for the generation of the core-type emission, and the Cherenkov-drift instability is responsible for the generation of the cone-type emission. These electromagnetic instabilities are the strongest instabilities in the pulsar magnetosphere (Lyutikov 1999). This differs from the more common case of a nonrelativistic plasma, where electrostatic Cherenkov-type instabilities (i.e., those that result in emission of electrostatic Langmuir-type waves) are generally stronger than electromagnetic instabilities. There are two main reasons for this difference. First, in a relativistic plasma, electromagnetic wave-particle interactions become as strong as electrostatic ones. In addition, for a one-dimensional plasma streaming along the magnetic field, the effective parallel mass, i.e., $m_{e f f \|}=\gamma^{3} m$ ( $m$ is the mass of a particle, and $\gamma$ is its Lorentz factor), is considerably increased by relativistic effects. This suppresses the development of the electrostatic instabilities. In contrast, the effective transverse mass, $m_{e f f \perp}=\gamma m$, is less affected by the large parallel momentum.

\footnotetext{
${ }^{1}$ Also at: Theoretical Astrophysics, California Institute of Technology, Pasadena, California 91125, U.S.A.
} 
For both instabilities, the energy is transferred from a particle to a wave through a resonant interaction. In plasma physics, such instabilities are also sometimes called "incoherent broadband masers". In pulsars, unlike atomic lasers where emission is generated only in a single narrow line, a particle can simultaneously resonate with several different waves in a broad bandwidth, but different amplified waves have random phases: they are incoherent.

The cyclotron instability can develop in both the primary beam and in the tail of the plasma distribution. The Cherenkov-drift instability develops on the rising part of the beam distribution function. The free energy for the growth of the instability comes from the nonequilibrium, anisotropic distribution of fast particles.

For the development of both instabilities, the following conditions should be satisfied: (i) the medium supports subluminous waves, i.e. its index of refraction is larger that unity, (ii) the center of the particle's gyration is moving along the magnetic field with a velocity larger than the phase speed of the normal modes, and (iii) the "inversion population condition" for the resonant waves holds. All of these conditions are satisfied for the pulsar plasma.

From the microphysical point of view, the emission process has more similarities with Cherenkov emission than with synchrotron or curvature emission. In the case of the Cherenkov-type process, the emission may be attributed to the electromagnetic polarization shock front that develops in a dielectric medium due to the passage of a charged particle with speed larger than the phase speed of waves in the medium. It is virtually a collective emission process. Interestingly, in a cyclotron-Cherenkov emission process, an emitting particle undergoes a transition $u p$ in Landau levels, so population inversion requires more particles in the lower levels - this is satisfied by the one dimensional distribution of particles in a pulsar magnetosphere.

The emission of a charged particle propagating in a medium with a curved magnetic field differs from conventional Cherenkov, cyclotron, or curvature emission and includes, to some extent, the features of each of these mechanisms. We have developed a formalism for considering the emissivity of a particle in a curved field in a medium (Lyutikov, Machabeli, \& Blandford 1999b). The resulting process may be called, to some extent, a coherent curvature emission.

Instabilities develop in a limited region on the open field lines. The size of the emission region is determined by the curvature of the magnetic field lines, which limits the length of the resonant wave-particle interaction. The location of the cyclotron-Cherenkov instability is limited to the field lines with large curvature, while the Cherenkov-drift instability occurs on the field lines with the radius of curvature limited both from above and from below. Both instabilities produce narrow pulses, though they operate at radii where the opening angle of the open field lines is large.

\section{References}

Lyutikov 1999, J. Plas. Phys., 62, 65

Lyutikov M., Blandford, R. D., \& Machabeli, G. Z. 1999a, ApJ, 512, 804

Lyutikov, Machabeli, \& Blandford 1999b, MNRAS, 305, 338 